\author{
THE TRANSPORT LINE BETWEEN \\ THE MAIN RING AND THE ENERGY DOUBLER \\ A.G. Ruggiero \\ July 30,1974
}

\title{
Introduction
}

In this paper we describe the transport line between the main ring (MR) and the energy doubler (ED). The transport line is designed on the basis of the following three assumptions:

1. The ED is located at the apex of the same tunnel which encloses the MR. The relative locations of the two rings are shown in Fig. I. The ED line is 3 feet to the inside and 4 feet above with respect to the MR Iine. Watching Fig. I the beam moves toward the page and the centre of the rings is to the right. The continuous outer curve is the cross section of the tunnel in a typical long-straight section, the dashed inner curve is the cross section of the tunnel in the bent section.

2. The beam has to be extracted from one long-straight section of the MR (BO) and injected in the next medium-straight section of the ED (BI7). The two rings are assumed to have the same identical lattice structure.

3. To keep the aisle-way in the tunnel as clear as we can it is advisable to extract the beam horizontally but displaced first vertically upward and second horizontally inward. The beam is then injected vertically.

To avoid problems associated with trapped flux at low fields, it is advisable to inject at an energy substantially higher than 
$100 \mathrm{GeV}$. On the other hand, the last two assumptions limit the maximum energy to about $200 \mathrm{GeV}$. For higher energy one has, at the same time, to transfer the beam from one long-straight section to the next long-straight section and to obstruct a good fraction of the tunnel with magnets. Thus, the transport line we are going to describe here, has been designed for the transfer energy of $200 \mathrm{GeV}$.

The transport line is divided in three parts:

(a) The beam is extracted from the MR and, with alternate series of horizontal and vertical bendings, transferrea to a line which is 2 feet and 7 inches inside and 3 feet above the MR line. In Fig. 1, A is the location of the IR line, B that of the ED line and $\mathrm{C}$ is the location of the intermediate line.

(b) The $\mathrm{C}$ Iine begins in correspondance to the first end of the first bending magnet after the long-straight section and terminates in correspondance of the first end of the fourth bending magnet before the next medium-straight section. of course the $C$ line runs parallel to the ED line at a distance of $I$ foot below and 5 inches to the outside.

(c) The beam, after having been bent first horizontally and then vertically, is finally vertically injected in correspondance to the first magnet after the medium-straight section.

In the following we shall describe the details of these three parts. The cross-sectional view of the beam pattern is shown by the dotted line in Fig. 1.

Extraction and Displacement

The line is shown in Fig. 2. It is about $102 \mathrm{~m}$ long. 
The various elements of the line are listed, in their natural succession, in Table $I$.

The two horizontal electrostatic setpa (ES) are identical to those presently used for the beam extraction. They are $3.0 \mathrm{~m}$ long each and have a field of $100 \mathrm{kV} / \mathrm{cm}$. The total deflection angle is of $0.3 \mathrm{mrad}$. The beam is bumped through the ES in the same way as it is done now. If the beam has to be extracted in the long straight section $B$, the following magnets are required:

- Extraction Position Bump Magnets at A46 and B17

- Vertical Extraction Magnets at A47 and BI8

- Extraction Angle - Bump Magnets at A44 and BIO

- Pinger at 148

Observe that, now, the septa are located at the inside, and the beam is supposed to reach the ES displaced $2.5 \mathrm{~cm}$ to the Inside. After the horizontal separation, the beam will go through the three MR bending magnets and the four MR quadrupoles. (The elements of the line which are shared with the MR regular lattice are dashed.) At the exit of the last quadrupole, the beam comes out displaced by $3.5 \mathrm{~cm}$ (to the inside) with an angle of about $0.3 \mathrm{mrad}$ with respect to the reference MR equilibrium orbit.

A string of five Lambertson magnets (LAM) follow to displace the beam vertically. Also, these magnets are similar to those presently used for the beam extraction. They are $3.2 \mathrm{~m}$ long and have a field of $7.8 \mathrm{kG}$. The total bending angle is $18.6 \mathrm{mrad}$. With reference to the MR equilibrium orbit, at the exit of the fifth Lambertson, the beam comes out displaced to the inside by $4.0 \mathrm{~cm}$ and still with an angle of $0.3 \mathrm{mrad}$, and displaced above by $15 \mathrm{~cm}$ and with an angle of $18.6 \mathrm{mrad}$. 
Next on the line, there are two groups of horlzontal bending magnets (BH). Each group is made of six identical magnets, namely same field $(B=13.0 \mathrm{kG})$ and same length $(3 \mathrm{~m})$. The first group bends the beam up, the second group bends the beam down. The total length of the string is of about $39 \mathrm{~m}$. Each group bends $35 \mathrm{mrad}$, and of course the overall bend is zero. The overall radial displacement is of $74 \mathrm{~cm}$, (to the inside). Thus, at the end of the last $B H$, with respect to the $E D$ line (the $B$ 11ne), the beam is displaced outward by $13.4 \mathrm{~cm}$ with zero angle, is still making a vertical angle of $18.6 \mathrm{mrad}$ and is displaced about $4 \mathrm{~cm}$ below.

Finally, the beam is brought up at the level of the $c$ line by two vertical bending magnets (BV), each about $3 \mathrm{~m}$ Iong and having a field of $20 \mathrm{kG}$.

To have an idea of the size of the magnetic gaps required, we give here the sagittae in the various magnets:
IAM
$0.6 \mathrm{~cm}$

$\mathrm{BH}$

$0.9 \mathrm{~cm}$

BV

$$
1.3 \mathrm{~cm} \text {. }
$$

A pessimistic guess of the beam emittances at $200 \mathrm{GeV}$ gives:

$$
\begin{aligned}
& \varepsilon_{\mathrm{v}}=1 \times 10^{-7} \pi \mathrm{m} \times \mathrm{rad} \\
& \varepsilon_{\mathrm{H}}=4 \times 10^{-7} \pi \mathrm{m} \times \mathrm{rad} \\
& \Delta \mathrm{p} / \mathrm{p}=2 \times 10^{-4} \quad(\text { total). }
\end{aligned}
$$

Even taking the largest values $\beta=100 \mathrm{~m}$ and $x_{p}=6 \mathrm{~m}$, at most the beam width is $1.5 \mathrm{~cm}$ and the beam height $0.6 \mathrm{~cm}$. In conclusion the cross section of the existing Lambertsons is, certainly, adequate. For the $\mathrm{BH}$ magnets we recommend a gap $5 \times 3 \mathrm{~cm}^{2}$ and for 
the BV magnets a cap $3 \times 5 \mathrm{~cm}^{2}$.

At the end of the extraction line there is a $1.9 \mathrm{~m}$ long quadrupole (QS) which replaces the doublet at the end of the long-straight section in the $\mathbb{R}$ lattice. This quadrupole should be used as matching point between the extraction line and the $C$ line.

\section{The Transport Line}

This is the $C$ line and essentially a replica, element by element, of the ED lattice. The dipoles should be set at the field of $9 \mathrm{kG}$ and the quadrupoles at the gradient of $120 \mathrm{kG} / \mathrm{m}$. The line can be made of all superconducting magnets.

Injection in the ED

Before the beam is injected in the ED a local horizontal inward bump is created with the apex in the proximity of the upstream end of the first bending magnet after the medium-straight section (B17). The four magnets to make the bump can be located at B13, $\mathrm{B} 15$ and B19, B22. The field should be enough for a maximum distortion of $2.5 \mathrm{~cm}$. The beam is injected vertically and horizontally displaced by $4.0 \mathrm{~cm}$ to the inside.

The injection channel is made of two strings of four vertical bending magnets each $3.465 \mathrm{~m}$ long and a field of $7.8 \mathrm{kG}$. Each string bends $16.1 \mathrm{mrad}$. The first string (upstream) bends up and the second stream (downstream), so that the overall bend is zero. The second group is made of Lambertsons.

The vacuum skin between the two halves of the last Lambertson is located halfway between the injected beam and the closed-orbit bump winch are thus separated by $1.5 \mathrm{~cm}$. The beam is injected 
when the tune of the ED is set to 19.25 so that a particle can go around four turns before hitting the vacuum skin of the magnet. Since the beam is $20 \mathrm{usec}$ long, the four bump magnets are up during the injection of all the beam. Once the tail of the beam is in, then the four magnets are discharged with a damping time of about $60 \mu \mathrm{sec}$.

During the discharge the closed orbit will move to the equiIfbrium orbit and the beam is trapped.

We cannot think of any other alternative way to inject a single turn in the ED, mainly because of the high energy of the beam $(200 \mathrm{GeV}$ ) and the short space avallable (the medium-straight section is only $15 \mathrm{~m}$ long).

The injection line is schematically shown in Fig. 3 . and the succession of the elements of the line are shown in Table II. The $C$ Ine ends at the downstream end of the regular quadrupole $Q D$. The following two magnets $(B B)$ are $3 \mathrm{~m}$ long each and bend the beam horizontally inward by $10.3 \mathrm{mrad}$ altogether. Their field is of about 12 kGauss but they should have an independent power supply for better control of the injection alignment. Next is a string of four vertical bending magnets, each $3.465 \mathrm{~m}$ long and having a field of $7.8 \mathrm{kG}$, followed by a string of four vertical bending Lamberison of the same characteristics. The first string bends the beam up, the second down. Finally the beam is injected in the first regular dipole of the ED, which is the first one downstream the medium-straight section.

Observe that the sagitta in all the magnets of the injection line is no more than $2 \mathrm{~mm}$. Thus the horizontal bending magnets (BB) can be made superconducting with the same aperture of the regular 
ED dipoles. Also the four vertical bending magnets (BUP) can be made superconducting with a bore of 2 inches in diameter. The four Lambertsons, of colirse, ars corventional magnets with a geometry similar to the ones presertily used in MR extraction line. Actually, since there is enough separation between the beam lines, the first two Lambertsons can be replaced by C-shaped magnets having roughly the same cross section of ones also presently used in the MR extraction line. 


\section{Table I}

Elements of the First Part of the

Transport Iine Between

Main Ring and Energy Doubler

\begin{tabular}{|c|c|c|}
\hline QFE & Quadrupole & \\
\hline S1 & Drift & $0.5 \mathrm{~m}$ \\
\hline ES & Electrostatic Septum (H) & 3.0 \\
\hline S2 & Drift & 0.2 \\
\hline ES & Electrostatic Septum $(H)$ & \\
\hline s3 & Drift & 2.59767 \\
\hline $\mathrm{B}$ & Bending ( $\mathrm{H})$ & 6.0706 \\
\hline 0 & Drift & 0.3048 \\
\hline$B$ & Bending ( $H)$ & \\
\hline 0 & Drift & \\
\hline $\mathrm{B}$ & Bending $(H)$ & \\
\hline 0 & Drift & \\
\hline QFL & Quadrupole & 1.31953 \\
\hline$A 1$ & Drift & 0.346202 \\
\hline QFL & Quadrupole & 1.31953 \\
\hline A2 & Drift & 1.77292 \\
\hline QDL & Quadrupole & 1.31953 \\
\hline A3 & Drift & 0.271018 \\
\hline$Q D$ & Quadrupole & 4.2672 \\
\hline S2 & Drift & \\
\hline LAM & Lambertson (V) & 3.2 \\
\hline
\end{tabular}


S2

LAM

S2

LAM

S2

LAM

S2

LAM

S4

BHF

S2

BHF

S2

BHP

s2

BHP

s2

BHP

S2

BHF

S2

BHM

S2

BHM

s2

BHM

S2
Drift

Lambertson ( $V$ )

Drift

Lambertson (V)

Drift

Lambertson (V)

Drift

Lambertson (V)

Drift

Bending ( $\mathrm{H}$ )

$0.488 \mathrm{~m}$

Drift

BendinE ( $\mathrm{H}$ )

Drift

Bending (iI)

Drift

Bending ( $\mathrm{H}$ )

Drift

Bending ( $H$ )

Drift

Bendirg (H)

Drift

Bending ( $\mathrm{H}$ )

3.0

Drift

Bending ( $\mathrm{H}$ )

Drift

Bending (H)

Drift 
BHM

S2

BHM

S2

BHM

S2

BV

S2

BV

S2

QS

0

B
Bending ( $\mathrm{H})$

Drift

Bending ( $\mathrm{H}$ )

Drift

Bending ( $\mathrm{H}$ )

Drift

Bending ( V)

Drift

Bending ( $V$ )

Drift

Quadrupole

Drift

Bending ( $\mathrm{H})$
$3.0 \mathrm{~m}$

1.9 


\section{Table II}

Elements of the Third Part of the

Transport Line Between

Main Ring and Energy Doubler

QD

00

$\mathrm{BB}$

S2

$\mathrm{BB}$

s9

BUP

S2

BUP

S2

BUP

S2

BUP

S8

LAM

S2

LAM

S2

LAM

S2

LAM

S2

B
Quadrupole

Drift

Bending ( $H$ )

Drift

Bending ( $\mathrm{H}$ )

Drift

Bending ( $V$ )

Drift

Bending (V)

Drift

Bending (V)

Drift

Bending (V)

Drift

Lambertson (V)

Drift

Lambertson (V)

Drift

Lambertson (V)

Drift

Lambertson (V)

Drift

Bending ( $H$ )

Drift
$2.1336 \mathrm{~m}$

2.1082

3.0

0.2

2.11

3.465

5.06 


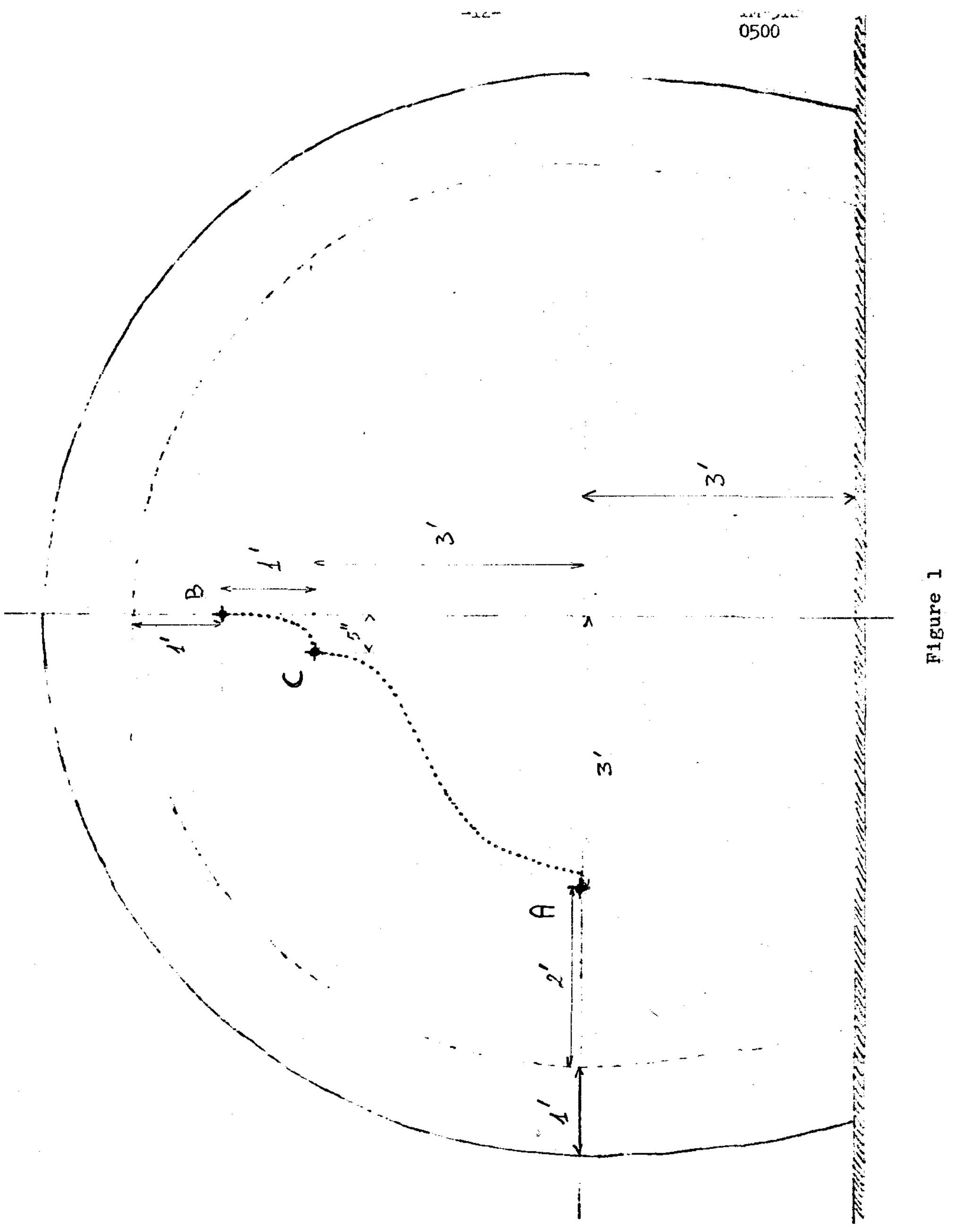




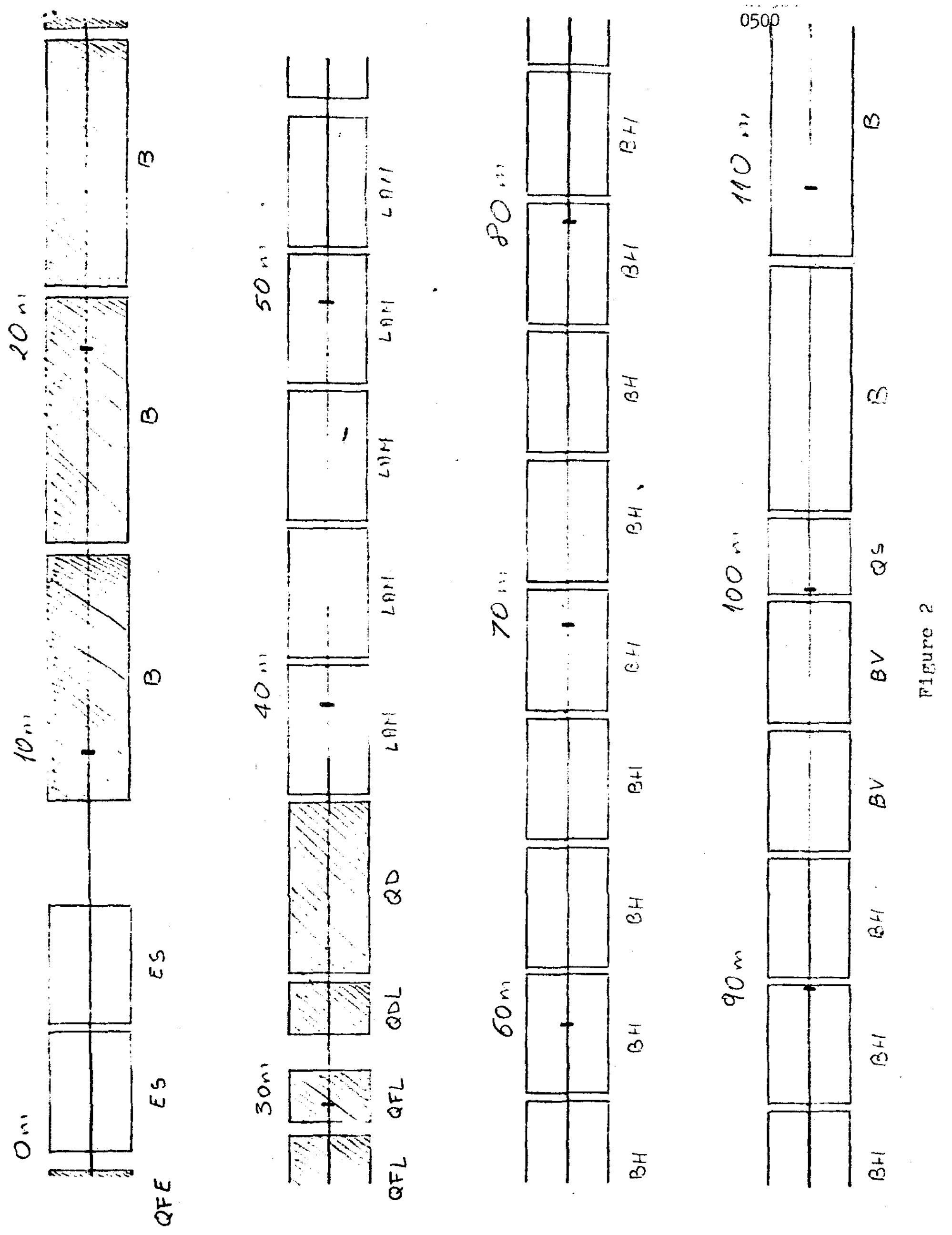




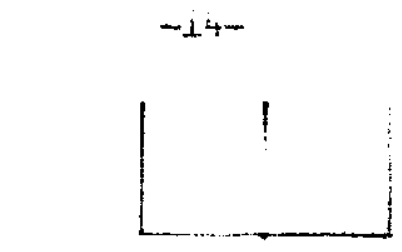

$24-512$
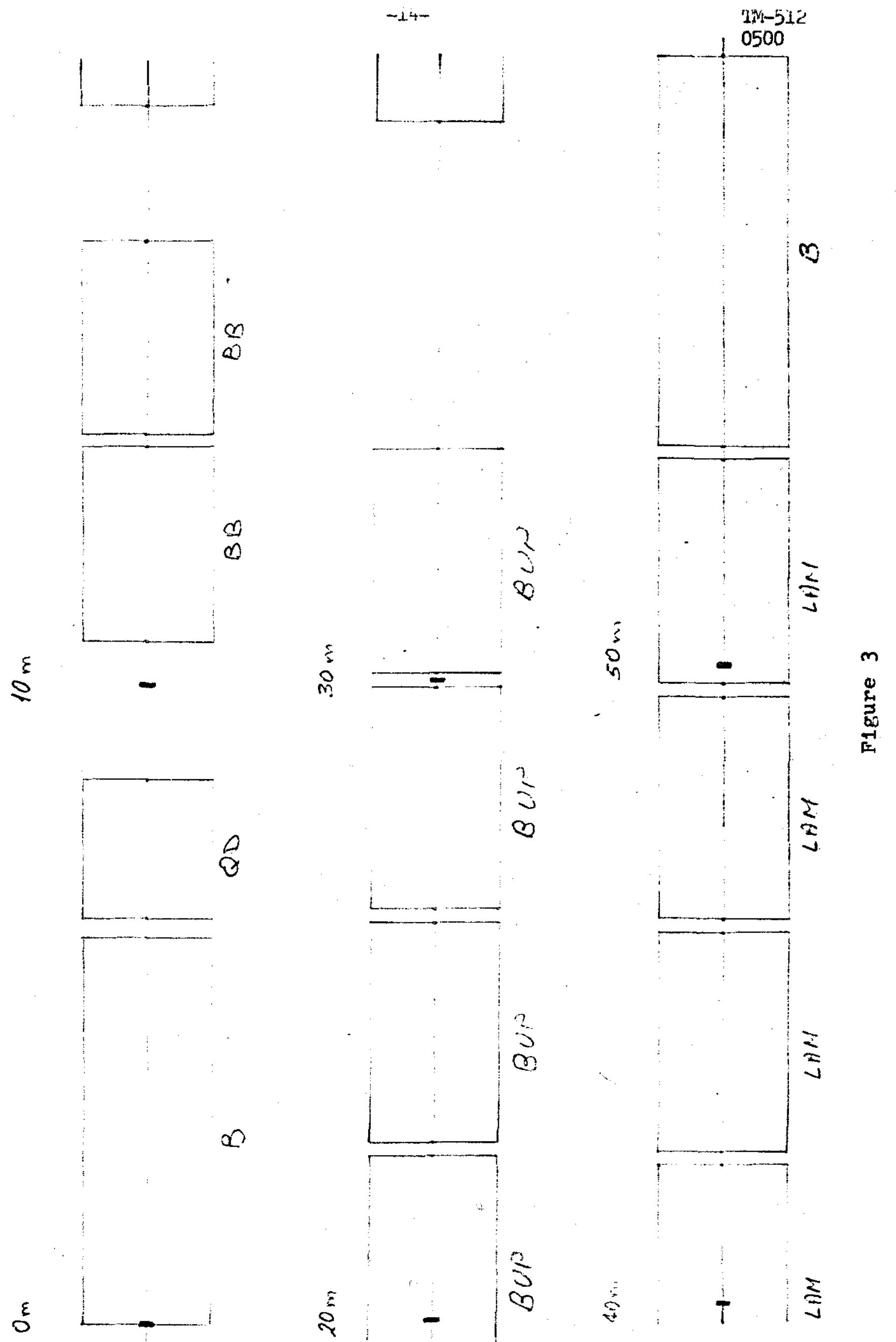